\title{
Tile TDAQ interface module for the Phase-II Upgrade of the ATLAS Tile Calorimeter
}

\author{
Xiaoguang Yue, on behalf of the ATLAS Tile Calorimeter System
}

\begin{abstract}
In order to meet the requirements for the High Luminosity-Large Hadron Collider (HL-LHC), a completely new architecture will be used to redesign the readout electronics of the ATLAS Tile Calorimeter (TileCal) system for the ATLAS Phase-II Upgrade. In the new Trigger and Data AcQuisition (TDAQ) architecture, the output signals of the Tile detector cells will be digitized in the front-end electronics and transferred for every bunch crossing to the off-detector Tile PreProcessor (TilePPr) modules through high-speed optical links. The TilePPr will then reconstruct energy deposited in each cell from the digitized samples and transfer the pre-processed cell energy data further to the Tile TDAQ interface (TileTDAQi) modules. The TileTDAQi will then group the cell energy data to generate trigger primitives with different granularity and implement interfaces based on different requirements from the Feature Extractor (FEX) and other trigger processor modules. At the same time, the TilePPr will also store the energy information in pipeline memories and send selected data out to the ATLAS data acquisition system through the TileTDAQi. The TileTDAQi module will be implemented on an Advanced Telecommunications Computing Architecture (ATCA) Rear Transition Module (RTM) format and will operate under the ATCA framework. In order to verify the basic functionality and interfaces of the TileTDAQi, a lightweight prototype has been designed and is now under test. This contribution will provide an overview of the new TileCal back-end electronics architecture and present the design and implementation of the TileTDAQi prototype together with preliminary test results.
\end{abstract}

Index Terms - High Energy Physics, ATLAS Tile Calorimeter, DAQ system, Field Programmable Gate Array, ATCA

\section{INTRODUCTION}

$T$ HE Tile Calorimeter (TileCal) [1] is the hadronic calorimeter installed in the central region of the ATLAS detector [2]. It performs precise measurement of hadrons, jets, taus and missing transverse energy, and provides information to the ATLAS trigger processors. As shown in Figure 1, the TileCal is divided into four cylindrical readout partitions: two Long Barrels (LBs) in the center and two Extended Barrels (EBs) on both sides. Each readout partition is composed of 64 wedge modules arranged in the $\varphi$ direction.

The TileCal is a sampling calorimeter utilizing iron as absorber and plastic scintillator as active material. When particles penetrate the plastic scintillator tiles, the generated scintillation light is collected by Wave Length Shifting (WLS)

Xiaoguang Yue is with the Kirchhoff-Institute for Physics, Heidelberg University, Im Neuenheimer Feld 227, D-69120 Heidelberg (e-mail: xiaoguang.yue@kip.uni-heidelberg.de).

Copyright 2019 CERN for the benefit of the ATLAS Collaboration. CCBY-4.0 license. fibers and transmitted to the PhotoMultiplier Tubes (PMTs) located at the outer edge of the TileCal modules.

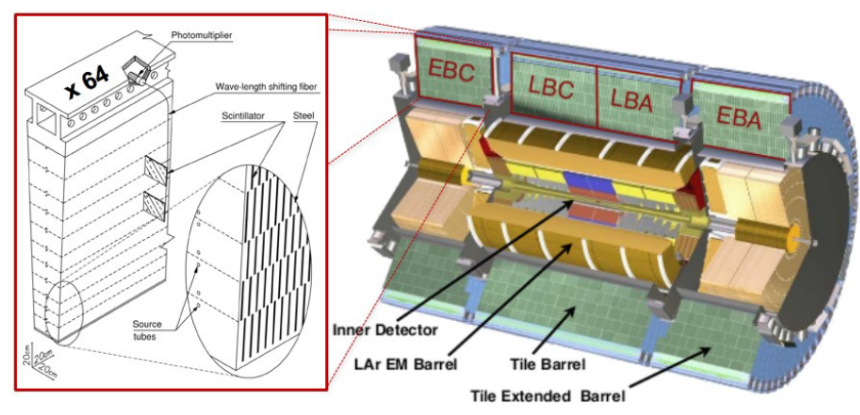

Fig. 1. A cutaway drawing of the ATLAS Tile Calorimeter together with a schematic of the wedge module. [3]

The Large Hadron Collider (LHC) will undergo a series of upgrades aiming at reaching $14 \mathrm{TeV}$ collision energy and increasing the instantaneous luminosity. After the Phase-II upgrade, the High Luminosity-LHC (HL-LHC) [4] will have an instantaneous luminosity 5-7 times larger than the nominal LHC value. As a result, the average number of proton-proton interactions per bunch crossing will be increased up to 200 . On the one hand, the higher luminosity means that there will be more interesting physical events for each bunch crossing; on the other hand, the higher event pileup condition and increased trigger rate will also present great challenges to the ATLAS detectors and the Trigger and Data AcQuisition (TDAQ) system.

In order to meet the requirements for the HL-LHC, the ATLAS TDAQ system will undergo a substantial upgrade. Accordingly, the TileCal on-detector and off-detector readout electronics will be completely redesigned using a new architecture for the ATLAS Phase-II upgrade [5].

\section{ThE TILECAl Phase-II UPGRADE}

The current readout electronics of the TileCal was designed about 25 years ago using the technology available at that time. As shown in Figure 2, after signal conditioning and amplification the PMT signals are digitized and stored in the pipeline memories implemented in the on-detector electronics. Only the event data selected by the Level-1 accept trigger signals are transferred to the off-detector Read-Out Driver (ROD) system. On the trigger path, cells in the same $\eta$ region are summed up to Trigger Towers (TTs) using dedicated analog circuits and then transferred to the offdetector Pre-Processor Modules (PPMs) [6], where the TTs are digitized and distributed further to the Level-1 Calorimeter Trigger (L1Calo) Processors [7]. 

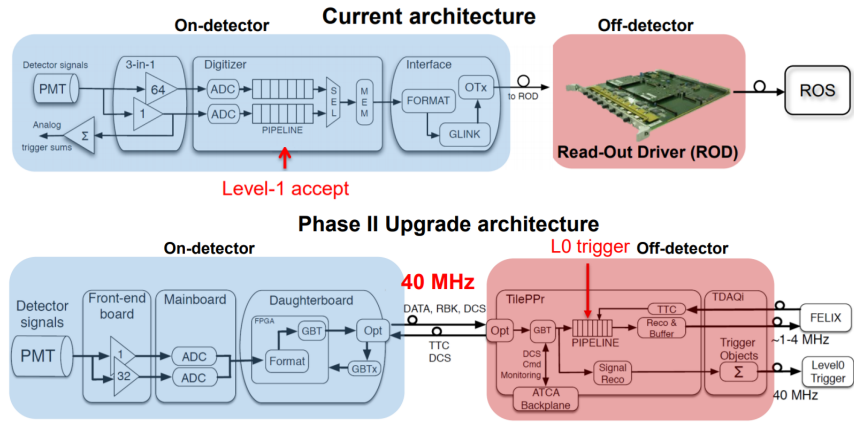

Fig. 2. The current TileCal readout architecture and the new readout architecture after the Phase-II Upgrade [10].

After the Phase-II upgrade, the TileCal readout architecture will look quite different. The new readout strategy is to transfer all digitized data from the on-detector electronics to the off-detector electronics through redundant optical links and to move the pipeline memories to the off-detector electronics. As a result, the readout throughput from the ondetector electronics to the off-detector electronics will increase from about $165 \mathrm{Gbps}$ in the current architecture to about 20 Tbps.

In order to reduce the system complexity and improve its flexibility, a concept of modular design based on the Advanced Telecommunications Computing Architecture (ATCA) is applied in the Phase-II TileCal off-detector electronics design. The block diagram of a final candidate design is shown in Figure 3.

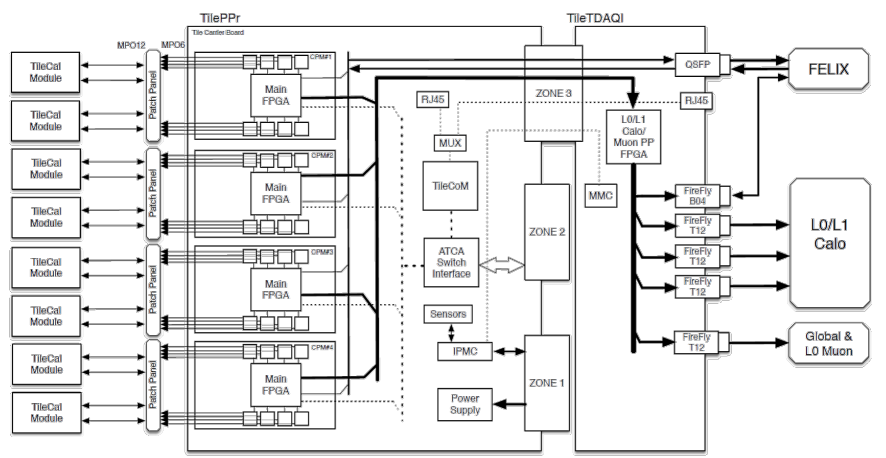

Fig. 3. Block diagram of a final candidate TilePPr and TileTDAQi design, a complete back-end system able to process the data from eight TileCal super-drawers. [10]

The Tile PreProcessor (TilePPr) module consists of one ATCA Carrier Base Board (ACBB) and four Compact Processing Modules (CPMs) mounted on the four AMC slots. The ACBB is a custom-designed ATCA-compliant carrier blade, which implements the main power distribution, Intelligent Platform Management Controller (IPMC) and the interconnections with the ATCA backplane and other submodules. The CPM is an Advanced Mezzanine Card (AMC), that it is compliant with ATCA and AMC standards. Each CPM communicates with two TileCal modules (1 LB and $1 \mathrm{~EB})$, it receives the digitized data sent from the ondetector electronics and stores them into pipeline memories waiting for a trigger decision. Upon receiving the Level-0 trigger signal, the relevant data will be transmitted to the Front End LInk eXchange (FELIX) [8] via the Tile TDAQ interface
(TileTDAQi) module. At the same time, the received digitized data are also used to reconstruct the energy deposited in each TileCal cell. The reconstructed cell energy data are sent from the CPMs to the TileTDAQi for every bunch crossing through electrical links. Besides that, the CPMs are also responsible for forwarding the LHC clock and Timing, Trigger and Control (TTC) commands from the FELIX to the TileCal ondetector electronics.

The TileTDAQi module is a custom-designed ATCA Rear Transition Module (RTM). It is responsible for interfacing the TilePPr with the FELIX and trigger processors. The TileTDAQi module receives the real-time cell energy data from the four CPMs connected to the same ACBB and then builds various trigger primitives with different granularity and different precision. Finally, the trigger primitives are distributed to the ATLAS Phase-II trigger system [9] through low- and deterministic-latency data links.

In the final system, there will be in total 32 TilePPr modules and 32 TileTDAQi modules installed in four ATCA shelves.

\section{THE TILETDAQi Module AND the First Prototype}

The TileTDAQi module is compliant with the ATCA RTM standard. It has a dimension of $322 \mathrm{~mm}$ in height and $92 \mathrm{~mm}$ in width, the maximum power consumption per module is $50 \mathrm{~W}$ for the final design.

The TileTDAQi connects with the ACBB using Zone-3 connectors. Three hard metric connectors from ERNI Electronics (type 973031) will be used to implement the $12 \mathrm{~V}$ power distribution from ACBB, the high-speed links to CPMs and all the slow control signals. The TileTDAQi will also include a Module Management Controller (MMC), which is responsible for the hot-swap power management, sensor reading, such as temperatures, voltages and currents to the ATCA shelf manager, and the communication with the IPMC on the ACBB module.

There will be in total six optical transceiver modules implemented on the TileTDAQi. One QSFP+ transceiver will be utilized to interface the four CPMs to the FELIX, it will provide one bidirectional link to each CPM module. The interface between the TileTDAQi processing FPGA and FELIX will be implemented using a bidirectional Samtec FireFly transceiver module. The other four 12-channel FireFly transmitter modules will be used to transfer trigger primitives from the processing FPGA to the trigger processors including the Level-0 Muon (L0Muon) trigger system, the electromagnetic Feature Extractor (eFEX), the jet Feature Extractor (jFEX), the global Feature Extractor (gFEX) and the Global trigger system.

One Xilinx Kintex Ultrascale FPGA will be used on the TileTDAQi board as the main processing unit. It will be responsible for the implementation of various high-speed data links with different data protocol and the data processing algorithms.

The functionality and the data flow that will be implemented in the processing FPGA is depicted in Figure 4. On the real-time path, the cell energy data from CPMs will be first decoded and aligned to the same bunch crossing. After 
that, the cell energies will be compared to two thresholds (one for the isolated muons and the other for the muon with a closeby jet) and the resulting energy flags will be sent to the L0Muon trigger system. In parallel, cells will be sorted (according to their energies) in descending order and those with energies greater than two times the noise level will be sent to the Global trigger system. At the same time, cell energies at the same $\eta$ region will be summed up into the TTs, each covering the $0.1 \times 0.1$ space in the $\eta-\varphi$ plane. After the data formatting, the TT information will be sent to the eFEX and jFEX modules, separately. The TTs will be further summed up into global Towers (gTs), each covering the $0.2 \times 0.2$ space in the $\eta-\varphi$ plane. The gTs will be sent to the gFEX module.

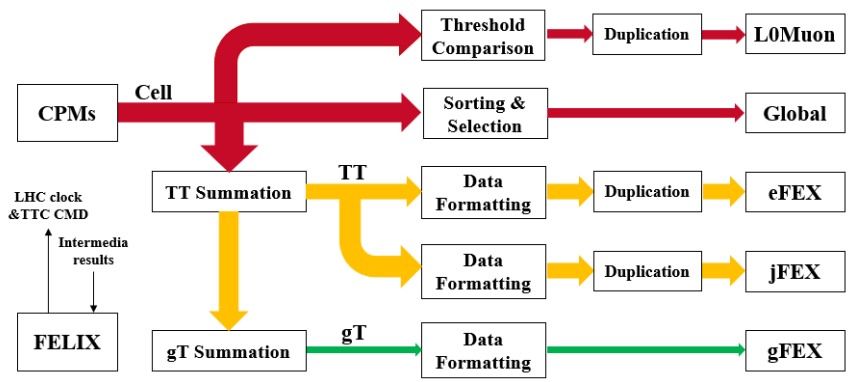

Fig. 4. The functionality of the TileTDAQi processing FPGA.

In addition, the FELIX interface will also be implemented to recover the LHC clock and decode TTC commands from the GBT data protocol; in the other direction, the TileTDAQi FPGA will also send some intermediate results on the readout path to FELIX in order to validate and monitor the trigger algorithms.

All interfaces and the processing logic on the TTC and readout paths must have a fixed and deterministic latency. All the high-speed links will be implemented using the Xilinx Multi-GigaBit Transceivers (MGT), and the reference clocks of the MGTs will be synchronized to the LHC clock recovered from the FELIX interface.

In order to validate and demonstrate the technical feasibility, the first TileTDAQi prototype has been designed by the Kirchhoff Institute for Physics at Heidelberg University. The prototype is a 16-layer Printed Circuit Board (PCB) using High-TG FR4 (TG170) as dielectric material. Compared to the final design, the whole infrastructure and a reduced version of connectivity are implemented in the prototype. The pin assignment of the Zone 3 connectors is designed to be compatible with both the custom designed ACBB and the AT8404 ATCA Carrier, which is a commercially available module from Kontron. This makes it possible to carry out preliminary system integration tests before the ACBB prototype is manufactured.

The placement of the main components on the prototype is shown in Figure 5. A CERN-MMC mezzanine card is utilized to communicate with the IPMC on the ACBB carrier via the Intelligent Platform Management Interface (IPMI).

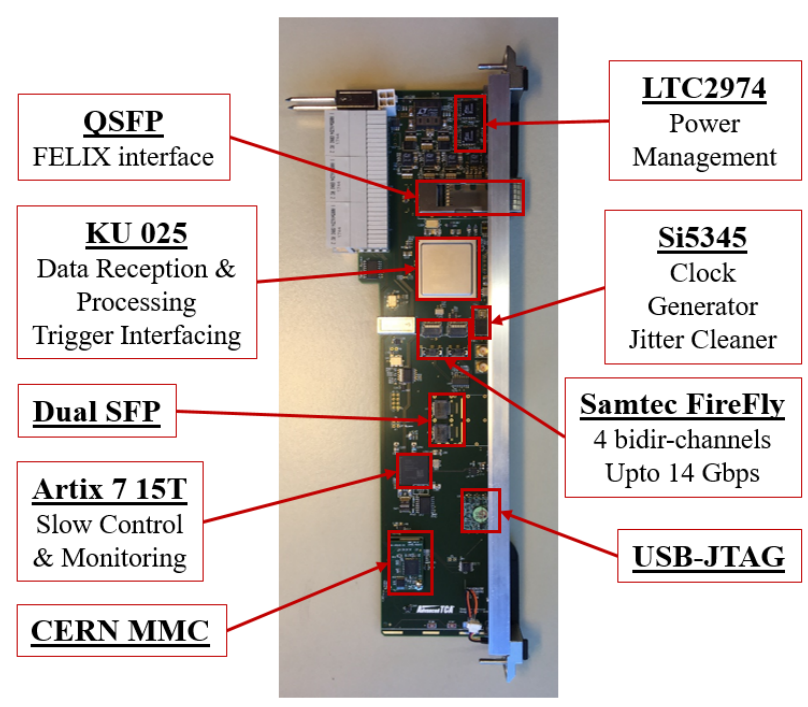

Fig. 5. The first TileTDAQi prototype and the placement of the main components.

An Artix-7 series FPGA from Xilinx is implemented as system controller. It is responsible for the slow control and monitoring of the peripheral components including power management modules, clock network components and optical transceiver modules.

The processing FPGA in the prototype is a Kintex Ultrascale KU025 device from Xilinx. The FPGA features 12 GTH MGTs and enough memory and logic resources to evaluate the data reception and transmission as well as the data processing algorithms.

On the lightweight prototype, interconnections to two AMC slots on the ATCA carrier, Slot 2 and Slot 4, are implemented. From each AMC slot, there are two bidirectional links connected to the TileTDAQi processing FPGA and two bidirectional links connected to the QSFP+ transceiver on the prototype. The links to the processing FPGA are used to validate the real-time data transmission from the CPMs to the TileTDAQi; whereas the links to the QSFP+ are used to validate the TTC path and the readout path between CPMs and FELIX.

There are also two Samtec FireFly transceivers and two $\mathrm{SFP}+$ transceivers on the prototype. The FireFly transceivers support link speed of up to $14 \mathrm{Gbps}$ and are used to validate the interfaces to the trigger processors; the $\mathrm{SFP}+$ transceivers, which support link speeds of up to $10 \mathrm{Gbps}$, are used to validate the interface between the TileTDAQi FPGA and FELIX.

\section{Standalone Tests ANd Preliminary System INTEGRATION TEST}

The first TileTDAQi prototype was assembled and ready for test in December 2018. Standalone tests have been carried out to verify the infrastructure functionality and test the link stability between the processing FPGA and the optical transceiver modules. In the standalone tests, the prototype is powered using an external $12 \mathrm{~V}$ power supply. The slow control interfaces on the system controller, an Artix-7 FPGA, 
and the functions of the power management modules, clock network components have been successfully tested and validated. The link stability between the processing FPGA and the optical transceiver modules was evaluated in optical loopback tests by using the Integrated Bit Error Ratio Test (IBERT) ipcore from Xilinx. The links to the SFP+ transceivers are tested with Pseudo-Random Binary Sequence31 (PRBS-31) at 9.6 Gbps; whereas the links to the FireFly transceivers are tested with PRBS-31 at 11.2 Gbps. Good link stability is achieved in both cases and the observed Bit Error Rate (BER) in the test was smaller than $10^{-15}$ for all the links.

Preliminary system integration has also been performed using the AT8404 ATCA Carrier, TilePPr Demonstrator (also known as sROD) [11] and the TileTDAQi prototype. The setup of the system integration is shown in Figure 6. The communication between the MMC on the TileTDAQi and the IPMC on the AT8404 has been successfully tested. The operation of TileTDAQi inside ATCA shelf has been validated as well.

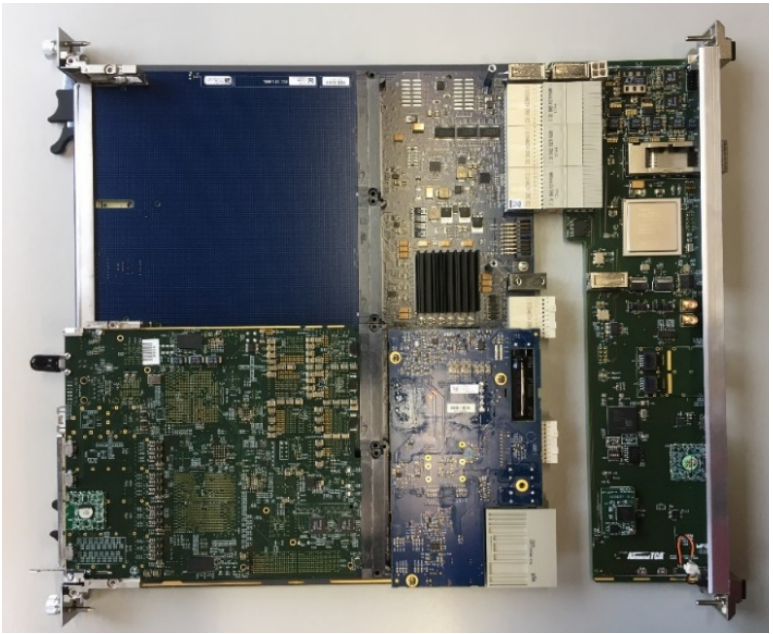

Fig. 6. The setup of preliminary system integration with AT8404 ATCA Carrier, TilePPr Demonstrator and TileTDAQi prototype.

The link stability between the TilePPr Demonstrator and TileTDAQi has been verified in the system integration test. The links on the TTC path (from the QSFP+ transceiver on the TileTDAQi to the TilePPr Demonstrator) are tested with PRBS-31 at $5 \mathrm{Gbps}$, and the observed link stability was quite good. For the links on the readout path (from the TilePPr Demonstrator to the QSFP+ transceiver on the TileTDAQi) and the real-time path (from the TilePPr Demonstrator to the processing FPGA on the TileTDAQi), degraded link stability was observed. On those links, no errors were detected when tested with PRBS-7 at $10 \mathrm{Gbps}$, but errors started to appear when tested with PRBS-31. The link stability degradation is probably caused by the long electrical traces on the AT8404 $(>30 \mathrm{~cm})$. In order to avoid similar problem, special routing optimization and post-layout simulation will be applied for the links on readout path and real-time path when designing the ACBB.

\section{Summary And OUTLOOK}

To meet the requirement imposed by the HL-LHC, the TileCal readout electronics will be completely redesigned for the ATLAS Phase-II Upgrade. The TileTDAQi module will become one of the core elements of the TileCal off-detector electronics, which provides the interface between the TileCal and the ATLAS TDAQ system.

The first TileTDAQi prototype has been developed and manufactured to validate the technical feasibility of the module design. The infrastructure functions have been successfully verified in standalone tests. A preliminary system integration test including AT8404 Carrier, TilePPr Demonstrator and TileTDAQi prototype has also been carried out and promising test results were achieved.

The first ACBB and CPM prototypes will be ready for test soon. The system integration test using ACBB, CPM and TileTDAQi prototypes is planned to be carried out in the first quarter of 2020.

\section{REFERENCES}

[1] ATLAS Collaboration, "Readiness of the ATLAS Tile Calorimeter for LHC collisions", Eur. Phys. J. C, vol. 70, no. 4, pp. 1193-1236, Dec. 2010.

[2] ATLAS Collaboration, "The ATLAS Experiment at the CERN Large Hadron Collider", JINST, vol. 3, S08003, Aug. 2008. DOI: $10.1088 / 1748-0221 / 3 / 08 / \mathrm{s} 08003$.

[3] Yue, X., "Data Acquisition and Software for the ATLAS Tile Calorimeter Phase-II Upgrade Demonstrator", EPJ Web Conf., vol. 214, 2019. DOI: https://doi.org/10.1051/epjconf/201921401004.

[4] Rossi, L. and Bruning, O., "High Luminosity Large Hadron Collider: A description for the European Strategy Preparatory Group", CERN, Geneva, Switzerland. CERN-ATS-2012-236, 2012. [Online]. Available: http://cds.cern.ch/record/1471000. DOI: $10.1140 /$ epjc/s10052-010-1508-y.

[5] ATLAS Collaboration, "Letter of Intent for the Phase-II Upgrade of the ATLAS Experiment," CERN, Geneva, Switzerland. CERN-LHCC2012-022; LHCC-I-023, 2012. [Online].

Available: https://cds.cern.ch/record/1502664.

[6] Asman, B. et al., "The ATLAS Level-1 Calorimeter Trigger: PreProcessor implementation and performance", JINST, vol. 7, P12008, Dec. 2012. DOI: $10.1088 / 1748-0221 / 7 / 12 / \mathrm{P} 12008$.

[7] Achenbach R. et al., "The ATLAS Level-1 Calorimeter Trigger", JINST, vol. 3, P03001, Mar. 2008. DOI: $10.1088 / 1748-0221 / 3 / 03 / \mathrm{p} 03001$.

[8] Anderson, J. et al., "FELIX: a High-Throughput Network Approach for Interfacing to Front End Electronics for ATLAS Upgrades", J.Phys.: Conf. Ser., vol. 664, no. 8, pp. 082050, Dec. 2015. DOI:10.1088/1742-6596/664/8/082050.

[9] ATLAS Collaboration, "Technical Design Report for the Phase-II Upgrade of the ATLAS TDAQ System", CERN, Geneva, Switzerland. CERN-LHCC-2017-020; ATLAS-TDR-029, 2017. [Online]. Available: https://cds.cern.ch/record/2285584.

[10] ATLAS Collaboration, "Technical Design Report for the Phase-II Upgrade of the ATLAS Tile Calorimeter", CERN, Geneva, Switzerland. CERN-LHCC-2017-019; ATLAS-TDR-028, 2017. [Online]. Available: https://cds.cern.ch/record/2285583.

[11] Carrio, F. et al., "The sROD module for the ATLAS Tile Calorimeter Phase-II Upgrade Demonstrator", JINST, vol. 9, C02019, Feb. 2014. DOI: $10.1088 / 1748-0221 / 9 / 02 / C 02019$. 